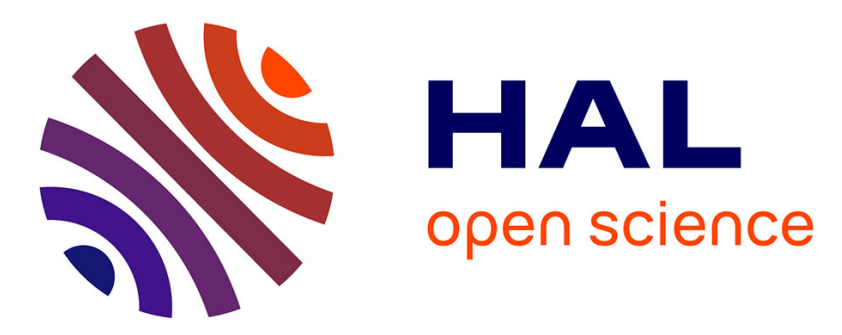

\title{
De-noising with wavelets method in chaotic time series: application in climatology, energy and finance
}

\author{
Dominique Guegan, Kebira Hoummyia
}

\section{To cite this version:}

Dominique Guegan, Kebira Hoummyia. De-noising with wavelets method in chaotic time series: application in climatology, energy and finance. Proceedings of SPIE, the International Society for Optical Engineering, 2005, 5848, pp.174 - 185. halshs-00180873

\section{HAL Id: halshs-00180873 \\ https://shs.hal.science/halshs-00180873}

Submitted on 22 Oct 2007

HAL is a multi-disciplinary open access archive for the deposit and dissemination of scientific research documents, whether they are published or not. The documents may come from teaching and research institutions in France or abroad, or from public or private research centers.
L'archive ouverte pluridisciplinaire HAL, est destinée au dépôt et à la diffusion de documents scientifiques de niveau recherche, publiés ou non, émanant des établissements d'enseignement et de recherche français ou étrangers, des laboratoires publics ou privés. 


\title{
DE-NOISING WITH WAVELETS METHOD IN CHAOTIC TIME SERIES: APPLICATION IN CLIMATOLOGY, ENERGY AND FINANCE
}

\author{
Dominique Guégan ${ }^{*, 1}$ kebira Hoummiya ${ }^{* *}$ \\ * ENS Cachan, MORA-IDHE UMR CNRS 8533 \\ guegan@ecogest.ens-cachan.fr \\ ** ENS Cachan, MORA-IDHE UMR CNRS 8533 \\ hoummiya@idhe.ens-cachan.fr
}

\begin{abstract}
In this paper, in order to de-noise a chaotic signal, we compare the time-frequency deconvolution method with the wavelets method. We apply our results on different dynamical systems and show the capability of wavelets' method to reconstruct the attractor of a chaotic time series. Then, we de-noise different data sets in order to re-built their attractor using the wavelets method. The applications concern temperatures and wind fluctuations, electricity spot prices and financial data sets.
\end{abstract}

Keywords: Attractor, Chaotic time series, Denoising, Wavelets method, real data.

\section{INTRODUCTION}

In this paper we discuss a new approach in order to reconstruct the attractor of a chaotic time series. The base of this problematic is related to the deconvolution of a noisy signal. This problem is not new and has been strongly documented in the literature. Here, we use an approach based on wavelets method. At the end of the 80's years, there was an increased interest for nonlinear systems having complex dynamics. At this time the techniques employed, to analyze the chaotic time series consisted mainly in calculating geometric and dynamical invariants of the underlying strange attractor. These quantities permit to quantify in some sense the chaotic behavior. At the same time a very interesting problem has been investigated by a lot of researchers. It concerns the deconvolution's problem. Indeed, the influence of any noise on data explained by chaotic systems is problematic, in one hand, for the reconstruction

\footnotetext{
1 Senior Academic Fellow de l'IEF, Directrice du Départe-
} ment d'Economie et Gestion. of these systems, in another hand for estimation theory useful to rebuilt all the invariants which characterize these systems and, finally in order to make predictions. A lot of approaches have been developed to try to bypass this problem. Most of them are based on noise-reduction methods. Other methods use global fit, for instance, the radial basis functions or the neural networks. To have a complete review on the chaos theory and the non-parametric methods adapted for the study of these systems, we suggest to read Lasota and Mackey (1994) and Guégan (2003), and references therein. In terms of deconvolution, the classical method in signal theory lies on the use of the Fourier transform. In praxis, we assume the frequency context of the signal to be stationary along the time axis. To overcome this restriction, researchers invented the windowed Fourier transform. This approach has shown its limits in terms to reconstruct chaotic time series. Here, we propose a time-scale analysis based on the wavelet method. Unlike the Fourier transform, the wavelet functions are localized both in time and in scale. 
They provide a convenient and efficient way of representing complex signals. To extract the signal which pollutes a system the wavelets method uses two different but complementary projections: one on an approximation space and the other one on the detail space. Since the wavelet transformation is an orthogonal operation, it preserves the probabilistic property of the underlying system and then, using this technique, we can expect to keep, apart from noise, all the useful information which characterizes a chaotic system when this one is highly polluted. The wavelets method is now well known, and we refer to Daubechies (1992) for a deep understanding on these techniques. In order to show the impact of the wavelets method on the reconstruction of the attractor of a noisy chaotic time series, we consider a dynamical system whose attractor presents specific properties. It is the Lorenz system represented by three equations:

$$
\left\{\begin{array}{l}
X_{t}=8.42\left(Y_{t-1}-X_{t-1}\right) \\
Y_{t}=X_{t-1}\left(45.92-Z_{t-1}\right)-Y_{t-1} \\
Z_{t}=X_{t-1} Y_{t-1}-4 Z_{t-1}
\end{array}\right.
$$

The paper is organized as follows. In Section two, we introduce some properties of the previous systems without noise and in presence of noise. In Section three, after a brief recall on the wavelets method, we show the efficiency of the Daubechies wavelets in the reconstruction of the de-noised Lorenz attractor. Applications on different real data sets are proposed in Section four.

\section{STATISTICAL PROPERTIES OF VARIOUS DYNAMICAL SYSTEMS:}

In this Section, we briefly presented the Lorenz system (1). We give its attractor in dimensions 2 and 3 , its trajectory, ACF, periodogram, histogram and we some statistics on the Figure ??. The trajectory of the Lorenz system appears quasi seasonal but it is not a true seasonality. We observe a slow decay of the autocorrelation function and almost one explosion on the periodogram. The histogram is bimodal. The kurtosis is not very high. For this system, we denote three different kinds of behaviors: existence of an attractor, a long memory behavior and existence of two states. This process is well known to be a dynamical chaotic system, and as a stochastic process, its behavior appears close to the behavior of long memory processes, (see Guégan, 2001). Now, we add a measurement noise on this system. Thus, if $\left(X_{t}\right)_{t}$ represents the original chaotic system, $\left(\varepsilon_{t}\right)_{t}$ any noise, this means that we observe a time series $\left(Y_{t}\right)_{t}$ which follows the recursive scheme, $\forall t$ :

$$
\left\{\begin{array}{l}
Y_{t}=X_{t}+\varepsilon_{t} \\
X_{t}=f\left(X_{t-1}\right)
\end{array}\right.
$$

With $f$ representing the Lorenz system. In a first step, we are interested by the changes observed
Figure 1. Graphics for the Lorenz system (1)
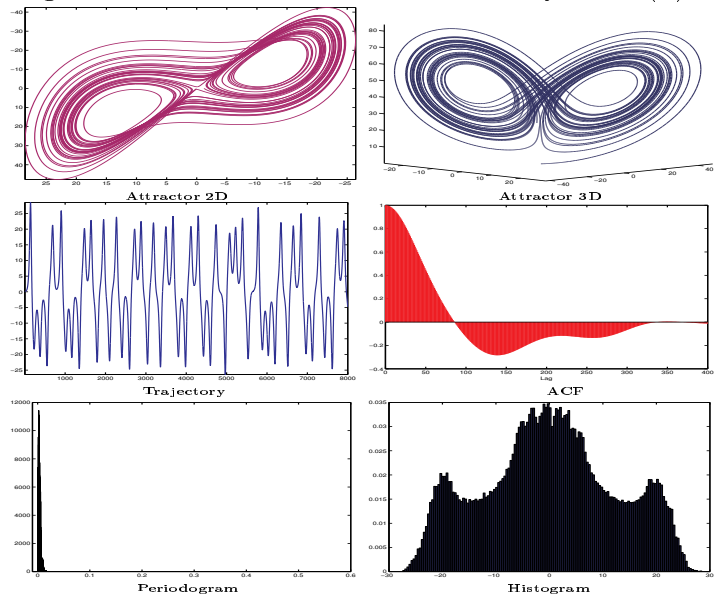

on the statistical properties of the noisy chaotic system and we compare them with the properties of the true chaotic system. In a second step, which is the main purpose of this work, we focus on the reconstruction of the de-noised attractor. Here, we add a Gaussian measurement white noise whose mean is equal to 15 and variance equal to 10 . On figure 2 we provide the attractor in dimension three. We observe that the Lorenz' attractor is not as visible as on the Figure ??. Nevertheless we can conjecture existence of such an attractor because the pollution is not too high. For this system, the autocorrelation function and the spectral density exhibit a behavior similar to the one observed on the Figure ??. The empirical distribution does not exhibit two modes and the kurtosis of the system is not very high. Now, we are interested by the reconstruction of the de-noised attractor and we use the wavelets method.

Figure 2. Polluted attractor in D3, with Gaussian noise, mean 15 and variance 10 .

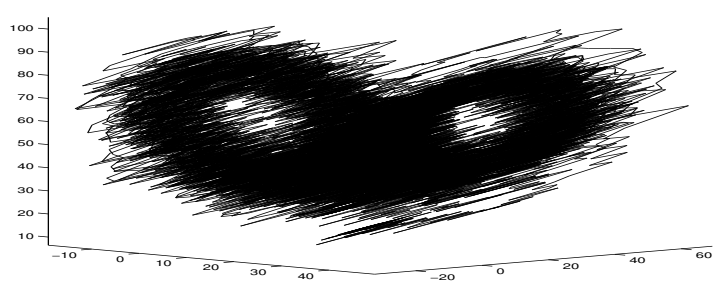

\section{WAVELET METHOD}

The fundamental uncertainty principle, which is the basis of a lot of filters like the high pass filter, the low pass filter, the medium pass filter, etc..., excludes absolute precision of information in the time and frequency domains simultaneously. This trade-off between information in the time and frequency domain inspires multi-resolution analysis. It is clear that a multi-resolution must be a form of smoothing. Daubechies (1992) provides an inspiring method based on the use of explicit orthonor- 
mal bases on multi-resolution analysis. Wavelets bases offer a degree of localization in space as well as in frequency that enables the decomposition of a signal into compactly supported oscillating components. The coefficients associated with each of the components are called wavelet coefficients. A remarkable property of wavelet coefficients is to reflect the local regularity of the original function, being large when the function is irregular and small when the function is smooth. The property is very useful to detect discontinuities or sharp changes in a noisy signal.

Wavelet coefficients are discrete transformations of a so-called mother wavelet $\psi$. First a doubly indexed family of wavelets is generated, by dilating and translating $\psi$,

$$
\psi_{j, k}(t)=2^{j / 2} \psi\left(2^{j} t-k\right),
$$

$j, k \in \mathbb{Z}$. The wavelet coefficient of a process $\left(Y_{t}\right)_{t}$ is a function of the scale parameter $j$ and translation parameter $k$ and is defined by:

$$
w_{j, k}=2^{j / 2} \int Y_{t} \psi\left(2^{j} t-k\right) d t .
$$

In the following, we will choose wavelet functions which are well located around zero (Daubechies functions), decreasing rapidly to zero as $t \rightarrow \infty$ and oscillating such that $\int \psi(t) d t=0$. We will strengthen these conditions imposing more vanishing moments $P$ and specific conditions we specify latter. The wavelet coefficients $w_{j, k}$ represent how much information is lost (gained) if the series $\left(Y_{t}\right)_{t}$ is sampled less (more) often. The index $j$ is called the resolution level and corresponds to a frequency $2^{-j}$, the index $k$ is called the time (or space) parameter and corresponds to the dyadic position $\frac{k}{2 j}$. For each level $j$, the detail signal is given by $\forall t: D_{j}=\sum_{k \in \mathbb{Z}} w_{j, k} \psi_{j, k}(t)$, and then the global reconstruction of the signal is equal to $Y_{t}=\sum_{j} \sum_{k \in \mathbb{Z}} w_{j, k} \psi_{j, k}(t)$. Now, when we fix a level $J$, we can get the approximation signal: $A_{J}=\sum_{j>J} D_{j}$, and the reconstruction of the true $\left(X_{t}\right)_{t}$ signal denoted $\left(\tilde{X}_{t}\right)_{t}$ is obtained as the sum of approximation signal at level $J$ and its finer details. When we separate the noise from the signal using the wavelets' theory, the noise appears in the detail coefficients. Thus, to remove this noise, we proceed in two steps: we need to determine a certain threshold which permits to keep the details which are interesting for the reconstruction: we denote this threshold $\lambda$ in the following. The choice of this threshold is very difficult and it does not exist, until now, an universal method to choose it. In the following, we use:

$$
\lambda=\frac{\left.\max \left(w_{j, k}\right)+\max \left(\mid w_{j, k}\right) \mid\right)}{2}
$$

When the choice of the threshold is done, we have to determine the thresholding function to threshold the wavelet coefficients. Two functions are mainly used in the literature : the soft thresholding one and the hard thresholding one, introduced by Donoho and Johnstone (1994). In the following we have used these two thresholding functions.

To show the effect of the wavelets method in denoising a system, we show how we are able to reconstruct the lorenz system when this one has been polluted as on Figure 2. We use Daubechies wavelets with soft and hard thresholding functions, the threshold $\lambda, \mathrm{J}=4$ and $\mathrm{P}=16$. The reconstructions are given on the Figure 3 . These reconstructions are particularly interesting because we are able to obtain the two states which characterizes this system and we can follow the signal passing from one state to another one. The values obtained for the MSE are quite small. To get this reconstruction the level of scale is not high, but in counter part we need to use a high number of vanishing moments.

Figure 3. De-noised attractor using Daubechies functions with $\mathrm{J}=4, \mathrm{P}=16$.

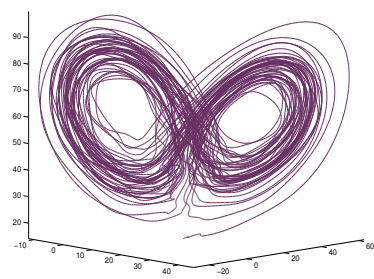

Hard-thresholding

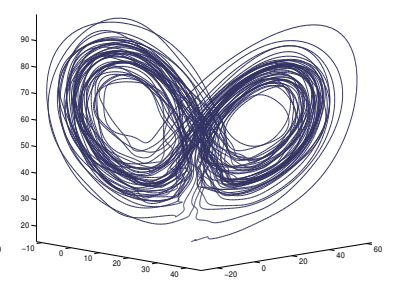

Soft-thresholding

\section{APPLICATIONS TO REAL DATA}

In this Section, we illustrate our previous approach using real data sets. For the different data sets we consider below, we only know their trajectory and we try to see if it is possible to associate to these data sets a pseudo-attractor. Here our approach has to be viewed like an explanatory exercise in order to reconstruct such attractor following the intuition given by Takens' theorem (1981) and using wavelets method. The data are respectively temperature fluctuations, wind speed, $\mathrm{CAC}_{40}$ index and electricity spot prices. For each data set, we specify the period on which we observe them, their frequency, their statistics. Then we specify how we embed them. We will see that different values of the delay $\tau$ are used with respect to each data set. We give the characteristics of the reconstruction of the pseudo- attractor via wavelets method.

\subsection{Climatology}

4.1.1. Daily temperature: The temperature fluctuations have an impact on business activities like power producers, energy consumers, tourism 
activity or agricultural industries, then it is important to get a good modelisation of this kind of fluctuations. Here, we study daily temperatures provided by the French Meteo Service to the laboratory IDHE (ENS Cachan). This chronic has been recorded in Paris (Montsouris Park) from the 1st of January 1873 to the 31st december 2002, thus the sample size is equal to $\mathrm{N}=47481$. The temperatures are in Celsius degrees and each data correspond to the maximum daily observations. We provide the trajectory, the autocorrelation function, the histogram and the periodogram for the full period under study on Figure 4. We observe that the trajectory is second order stationary, its autocorrelation function does not decrease towards zero very quickly, its periodogram explodes in a frequency which is far from zero and its histogram presents two modes. If we modeled the series using a stochastic process, we probably try to adjust some long memory process but existence of long memory is not contradictory with existence of an attractor, see Guégan (2001) and (2003). To reconstruct the possible attractor

Figure 4. Graphics and statistical properties for fluctuations temperatures recorded in Paris (Montsouris Park) from the 1st of January 1873 to the 31st december 2002 .
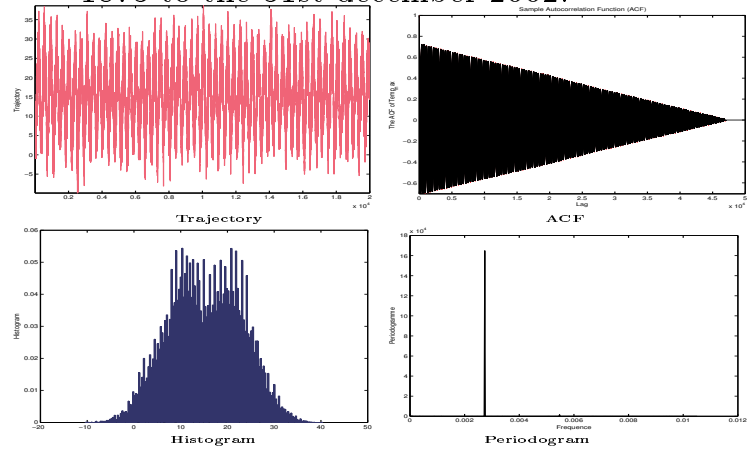

characterizing this data set, we embed the data $\left(Y_{t}\right)_{t}$ represented on the Figure 4, in the spaces $\left(Y_{t}, Y_{t-1}\right)$ and $\left(Y_{t}, Y_{t-76}\right)$ and we provide the two scatter plots on the Figure 5. The two scatter plots appear completely different. On the Figure 5 (1), we cannot suspect existence of an attractor. The representation appears close to the representation of a non stationary process. Thus, it seems more interesting to consider the representation given on the Figure 5 (2), to try to re-built a pseudo - attractor. In that latter case this means that we are going to reconstruct the attractor using data recorded every 2 months and half. Using Daubechies wavelets, we provide the reconstruction using Daubechies wavelets with $J=7$ and $P=15$, on the Figure 6 . To get more information concerning this reconstruction, we need now to compute the Lyapunov exponents and to make forecasting. This will done in another paper.
Figure 5. Embedding of the maximum fluctuations temperatures recorded in Paris from the 1st of January 1873 to the 31st december 2002

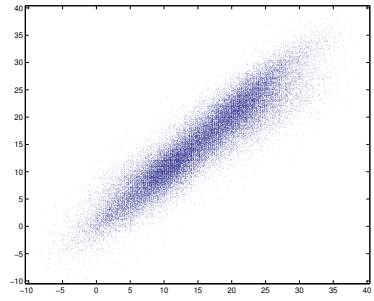

$\left(Y_{t}, Y_{t-1}\right)$

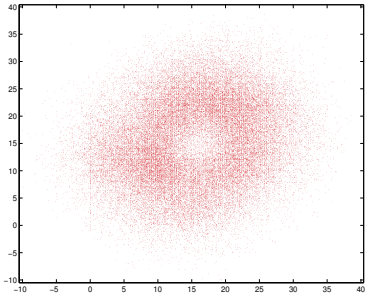

$\left(Y_{t}, Y_{t-76}\right)$
Figure 6. Building of the pseudo attractor for maximum fluctuations temperatures using Daubechies wavelets with $\mathrm{J}=7$ and $\mathrm{P}=15$.

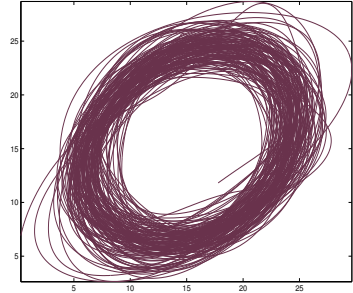

Hard-thresholding

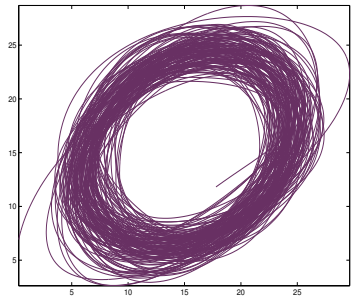

Soft-thresholding
4.1.2. Wind speed There is not many works on the modelling of wind speed. Knowing such modelisation is important, in particular for insurance companies. Here, we investigate the wind speed data collected every 3 hours UTC in $\mathrm{m} / \mathrm{s}$, at Montsouris Park in Paris. They have been provided by the French Meteo Service to the laboratory IDHE (ENS Cachan). The data are observed from the 17th of December 1996 at 9:00 a.m to the 30th November 2004 at 9:00 p.m. The sample size of the data set is $N=23245$. The data appear second order stationary. The autocorrelation function does not decrease towards zero very quickly and the periodogram explodes in one frequency far from zero. Thus, these data seem to exhibit a long memory behavior in the covariance sense, see Guégan (2004). Now, the histogram is asymmetric and looks like a Log-normal distribution. When we embed the data in the spaces $\left(Y_{t}, Y_{t-1}\right)$ and $\left(Y_{t}, Y_{t-8}\right)$, we obtain the figure $(7)$.

Figure 7. Embedding of the wind speed data recorded from the 17th of December 1996 to the 30th November 2004.

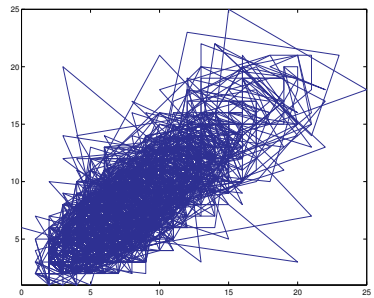

(A) $\left(Y_{t}, Y_{t-1}\right)$ : lag 3 hours

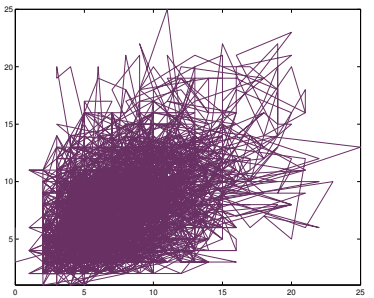

(B) $\left(Y_{t}, Y_{t-8}\right)$ : lag one day
We can distinguish some organization inside these two clouds. It seems that the orbits belong to 
different ellipses whose diameters change all the time. This appears more and more clearly when $\tau$ grows up, in particular in the space $\left(Y_{t}, Y_{t-8}\right)$, which corresponds to a daily data set. In order to find an attractor which can explain the structure which governs these data, we de-noise the two data sets using Daubechies wavelets with $J=$ 4 and $P=13$. The speudo-attractors can be observed on the figure 7 . Their structure is quite different.

Figure 8. De-noising wind speed data, using daubechies wavelets with $\mathrm{J}=4$ and $\mathrm{P}=13$.

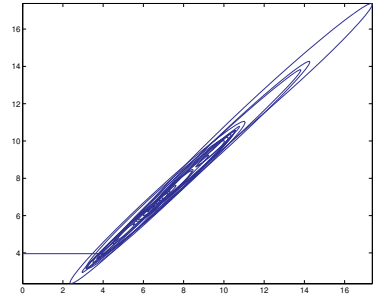

Pseudo attractor of (A): $\left(\tilde{X}_{t}, \tilde{X}_{t-1}\right)$

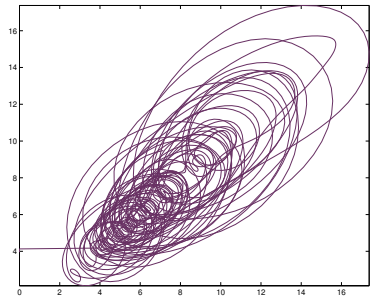

Pseudo attractor of (B): $\left(\tilde{X}_{t}, \tilde{X}_{t-8}\right)$

\subsection{Financial data}

In this Subsection, we analyse a financial data set: The $\mathrm{CAC}_{40}$ which is an intra-day data set from database of Euronext. The detection of possible attractors inside financial data sets is not new. A lot of researchers have already try to detect attractors inside financial data sets. We investigate here the intra-day index $\mathrm{CAC}_{40}$ of the French stock exchange during the month January 2004. The quotations, denoted $\left(Y_{t}\right)_{t}$, are recorded every 30 seconds during working days in a week between 9:00 a.m. to 5:30 p.m., then we have 21322 observations. We observe that the data are not second order stationary. There exists a trend: this explains the form of the autocorrelation function and of the periodogram. The form of the histogram also confirms the existence of two states inside the data, which corresponds to two distinct levels.

We make the data stationary using the returns $\left(r_{t}\right)_{t}$ defined $\forall t$, by $r_{t}=Y_{t}-Y_{t-1}$. We choice to work with returns taken every 3 minutes and 30 seconds and we plot them on the left of the Figure 9. This corresponds to the cloud $\left(r_{t}, r_{t-7}\right)$. In order to find the pseudo-attractor which characterizes these data, we de-noise this cloud using Daubechies wavelets with $J=6$ and $P=20$ and we get the attractor given on the Figure 9 on the right. The result is not surprising and confirms the results already mentioned in a lot of papers.

\subsection{Energy: Electricity spot prices}

We study in this Subsection, electricity spot prices. The modelization of electricity spot prices
Figure 9. Intra-day $\mathrm{CAC}_{40}$ recorded on the month january 2004, every 3 minutes and 30 second.

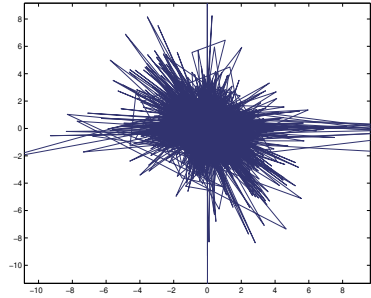

Embedding on $\left(r_{t}, r_{t-7}\right)$

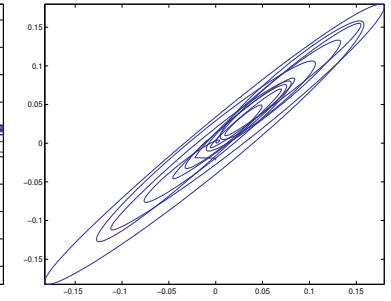

Pseudo attractor, $\mathrm{P}=20 \mathrm{~J}=6$ becomes recently a crucial issue for a lot of institutions. They enter in different kinds of commodities and the knowledge of their behavior is very important. The data under study correspond to the spot prices of the German market. The data have been obtained from the European Energy Exchange. They correspond to hourly prices from 16th of June, 2000, to the 16th of December, 2004. The sample size is $N=39480$. These data present strong heteroscedasticity and also very important explosions. This kind of data set is well know for existence of explosions as we can observe on the trajectory. These points increase the kurtosis. For this data set, we represent the scatter plot $\left(Y_{t}, Y_{t-12}\right)$ on the Figure 10 (a). This cloud suggests existence of some organization inside the data: it is not convex, and two states are visible. Thus, using 12-hours data set, we de-noise the data using Daubechies wavelets with $J=5$ and $P=8$. On the Figure 10, we provide the reconstruction of the speudo-attractor.

Figure 10. Reconstruction of the pseudo-attractor for electricity spot prices using Daubechies wavelets with $\mathrm{P}=8$ and $\mathrm{J}=5$.

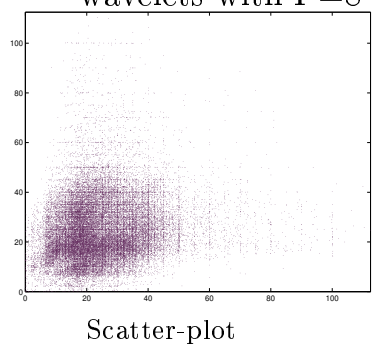

$\left(Y_{t}, Y_{t-12}\right)$

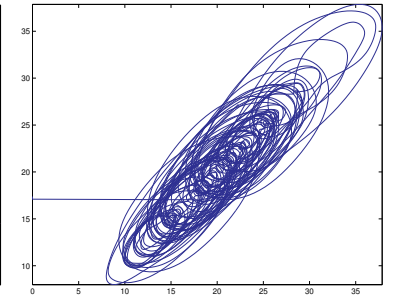

Pseudo-attractor: $\left(\hat{X}_{t}, \hat{X}_{t-12}\right)$
In summary, all these data sets present features which correspond to the characteristics of a lot of stochastic processes like the GARCH process, the Markov switching process, the SETAR process and the long memory process. Thus it seems important to study these data sets from a stochastic point of view. In another hand, we have shown in this paper that it is also possible to extract from these data some pseudo-attractor: we need now to investigate the properties of these pseudo - attractors. This will be done in another paper and the "chaotic" approach will be compared with the stochastic processes, via forecasting methods. 
Details concerning all these data can be found in Guégan and Kebira (2005).

\section{CONCLUSION}

In this paper we have showed that when a chaotic dynamical system is polluted by a noise, the attractor disappears. We show that the wavelets' method permits to de-noise such polluted models and permits to reconstruct the original attractor with very nice precision. The methodology developed here appears new in order to de-noise a chaotic system and gives very interesting results. We apply this approach on different data sets like fluctuations temperature, wind' speed, spot electricity prices, $\mathrm{Cac}_{40}$ and Dow Jones. For each data set, using their specific properties, we determine in what space it is judicious to embed the data, then using Daubechies wavelets, we extract the pseudoattractor associated to these data. It leaves to study the properties associated to these different attractors, to make forecasts and to compare them with stochastic parametric methods. This is the purpose of another paper and it is not discussed here.

\section{REFERENCES}

[1] Bosq D. and Guégan D. (1995): "Nonparametric estimation of the chaotic function and the invariant measure of a dynamical system", Statistics and Probability Letters, 25, 201-212.

[2] Brock W.A (1988): "Distinguishing random and deterministig systems", Journal of Economic theory, 40, 168-195.

[3] Casdagli M. (1989): "Nonlinear prediction of chaotic time series", Physica D, 35, 335-356.

[4] Cohen E., Daubechies I., Vial P. (1993), "Wavelet bases on the interval and fast algorithm", Applied and Computational Harmonic Analysis, 1,54-81.

[5] Daubechies I. (1992): "Ten Lectures on Wavelets", volume 61 of CBMS-NSF Regional Conference Series in Applied Mathematics. SIAM, Philadelphia, 1992.42.

[6] Diongue A.K, Guégan D. and Vignal B. (2004): "Forecasting electricity spot market prices with a k-factor GIGARCH process", preprint MORA/IDHE/ 14-2003 ,Décenbre 2003.

[7] Eckmann J.P. and Ruelle D. (1985): "Ergodic theory of chaos and strange attactors", Review of Modern Physics, 57(3), 617-656.

[8] Farmer J.D. and Sidorowich J.J. (1988): "Exploiting chaos to predict the future and reduce noise", Preprint, 1988.

[9] Ferrara L., Guégan D. (2001): "Comparison of parameter estimation methods in cyclical long memory time series", in Developments in Forecast Combination and Portfolio Choice, eds. C. Dunis, J. Timmerman, Chapitre 8, 179-195, J. Wiley .

[10] Grassberger P. and Procaccia I. (1983): "Characterisation of strange attractors", Physical Review Letters, 50, 346.

[11] Grassberger P. and Schreiber T. (1991): "A simple noise-reduction method for real data", Physical Letter A, 160, 411-418.

[12] Guégan D. and Mercier L. (1998b): "Stochastic and chaotic dynamics in high frequency financial data: nonlinear modeling", eds. C. Dunis and B. Zhou, John Wiley and Sons, 87-107.

[13] Guégan D. (2001): "Long memory behavior for simulated chaotic time series", IEICE, Trans.Fondamentals, E 84-A , 2145-2154.

[14] Guégan D. (2003): "Les Chaos en finance, Approche statistique", 450p., Economica, Paris.

[15] Guégan D.and Vanharen M-L.(2003): "Deconvolution of the chaotic maps defined on $[0,1]$ polluted by measurement noise, using wavelets", preprint MORA/IDHE/ 2-2003 ,September 2003.

[16] Härdle W., Kerkyacharian G., Picard D. and Tsybakov A. (1998): "Wavelets, Approximations and Statistical Applications", Lecture Notes in Statistics, Springer.

[17] Kavasseri R.G. and Nagarajan R. (2005): "A multifractal description of wind speed recorders", Journal chaos, solitions and fractals, 24(1), 165-173.

[18] Kostelich E. J. and Schreiber T. (1993): "Noise reduction in chaotic time series data : A survey of common methods", Physical Review E, 48(3), 1752-1763.

[19] Lasota A. and Mackey M. (1994): "Chaos, fractals and noise : Stochastic aspects of dynamical systems", Springer-Verlag, New York.

[20] Lorenz E.N (1969): "Atmospheric predictability as revealed by naturally occurring analogues", J.Atmos.sci, 26 , 636-646 .

[21] Mallat S. (1998): "A Wavelet Tour of Signal Processing", Academic Press, SanDiego, 1998.43.

[22] Meyer Y. (1994): "Les ondelettes : algorithmes et applications", Armand Colin,Paris, 1994.

[23] Sauer T. (1992): "A noise reduction method for signals from nonlinear systems", Physica $D$, 58, 193-201.

[24] Takens F. (1981): "Detecting strange attractor in turbulence, in dynamical systems and turbulence", eds D.Rand and L.Young, Berlin:Springer.

[25] Todd Ogden R. (1997): "Essential Wavelets for Statistical Applications and Data Analysis", Birkhaüser, Boston, 1997.

[26] Tong H. (1990): "Nonlinear systems: a dynamical approch", Oxford Publ. 